\title{
Pulmonary valve reconstruction after annular augmentation in severe forms of tetralogy of Fallot
}

Vladimiro Vida, MD, PhD, Alvise Guariento, MD, Nicola Pradegan, MD, and Giovanni Stellin, MD, Padua, Italy

\footnotetext{
From the Pediatric and Congenital Cardiac Surgery Unit, Department of Cardiac, Thoracic, Vascular Sciences, and Public Health, University of Padua, Padua, Italy.

Disclosures: Authors have nothing to disclose with regard to commercial support.

Received for publication Oct 5, 2018; revisions received Dec 28, 2018; accepted for publication Jan 18, 2019 available ahead of print June 10, 2019.

Address for reprints: Giovanni Stellin, MD, Pediatric and Congenital Cardiac Surgery Unit, Department of Cardiac, Thoracic, Vascular Sciences, and Public Health, University of Padua, Via Giustiniani, 2, 35100 Padua, Italy (E-mail: giovanni.stellin@unipd.it).

J Thorac Cardiovasc Surg 2019;158:e53-5

$0022-5223 / \$ 36.00$

Copyright (C) 2019 Published by Elsevier Inc. on behalf of The American Association for Thoracic Surgery

https://doi.org/10.1016/j.jtcvs.2019.01.069
}

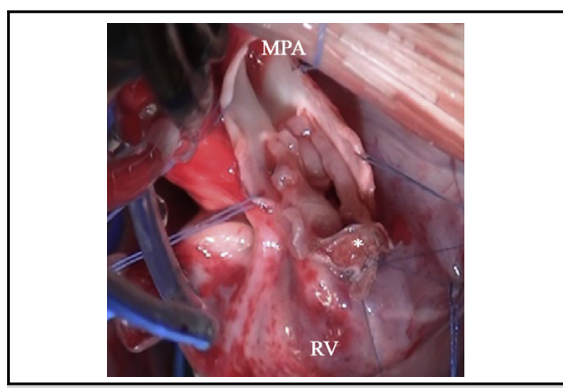

Reconstructed bicuspid PV after annular patch $\left(^{*}\right)$ augmentation. MPA, Main pulmonary artery; $R V$, right ventricle.

Central Message

The native PV can be preserved even in patients with ToF and marked PV annulus hypoplasia by combining PV annulus patch enlargement and complex PV plasty maneuvers.

See Commentary on page e57.
During the past decade, our institutional policy included the application of valve-sparing techniques during early repair of tetralogy of Fallot (ToF), ${ }^{1-4}$ with the aim of preserving long-term pulmonary valve (PV) and right ventricular function. We evaluated the results of PV preservation in patients with severe forms of ToF with marked PV annulus hypoplasia.

\section{SURGICAL TECHNIQUE}

A review of medical records and the Clinical Investigation Committee of the University Hospital of Padua approved computerized hospital data, and the procedures followed were in accordance with the Institutional Guidelines for retrospective record review and protection of patient confidentiality (Protocol n. 3254/AO/14). Patients were not identified, and the chairperson of the Ethics Committee consented for their data to be submitted for publication.

Surgical techniques aimed at preserving the PV during early repair of ToF have been described. ${ }^{1-3}$ In patients with PV Z-score 4 or less, the PV annulus is incised and the incision is prolonged for 3 to $4 \mathrm{~mm}$ into the subannular zone (Figure 1, A). A shallow biologic patch is used to enlarge the PV annulus circumference (to reach a PV annulus Z-score of 0 ) (Figure 1, B). The PV coaptation area is then extended (to cover the new pulmonary annulus area) by careful leaflet delamination ${ }^{4}$ (Figure 1, $C$ ). Subsequently, the extended cusps are resuspended directly onto the neo-PV annulus, thus creating a new PV commissure. The PV cusps occasionally need to be extended by using tiny triangles of prosthetic patch material (autologous pericardium) before resuspension (Figure 1,D). During repair, particular care is taken in removing any possible right ventricular outflow tract (RVOT) obstruction by combining transatrial and transpulmonary muscle band resection. Median cardiopulmonary bypass and crossclamp times were 141 minutes (interquartile range [IQR], 133-174) and $92 \mathrm{mi}-$ nutes (IQR, 88-101), respectively. A postoperative right ventricular pressure value greater than $50 \%$ of the systemic pressure is accepted in the absence of significant residual subpulmonary obstruction on 2-dimensional transesophageal echocardiography (Video 1).

\section{OUTCOMES}

Since August 2013, 12 patients with a median preoperative PV Z-score of -4.6 (IQR, -5.0 to -4.1 , with a lower value of -6.1 ) underwent early transatrial ToF repair. The PV was bicuspid in 8 patients $(67 \%)$, tricuspid in 2 patients $(17 \%)$, and unicuspid in 2 patients $(17 \%)$. The PV was dysplastic in 8 patients $(67 \%)$.

The median age at surgery was 3.9 months (IQR, 2.95 months). All patients underwent a PV annulus patch augmentation and PV leaflet delamination and resuspension 


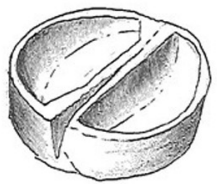

A

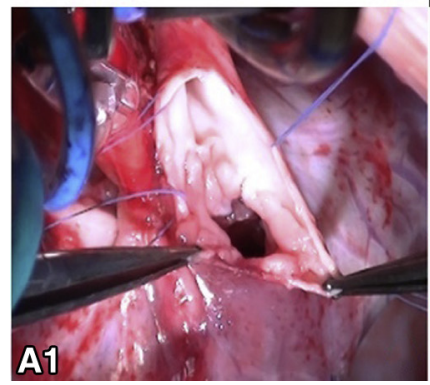

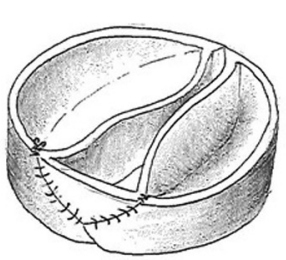

B

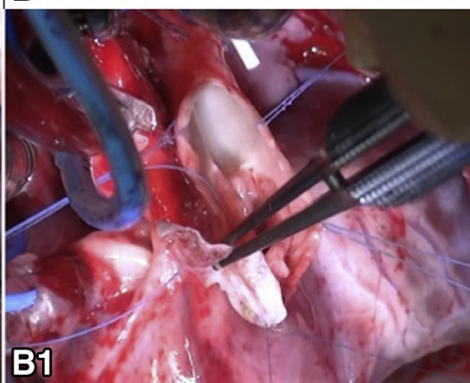

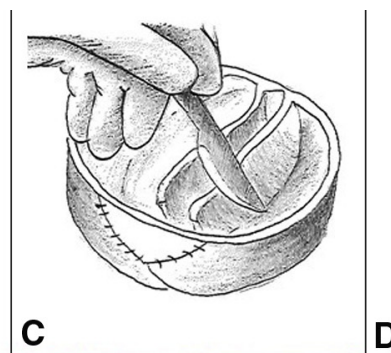

D
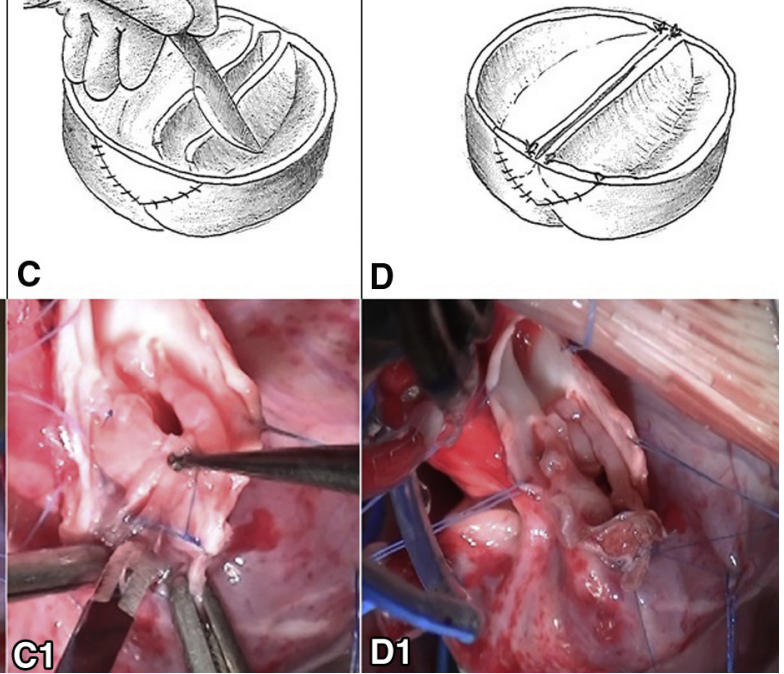

FIGURE 1. Intraoperative and matched images showing (A-A1) annular incision in patient with a bicuspid PV, (B-B1) PV annulus patch augmentation, (CC1) PV leaflet delamination plasty, and (D-D1) PV leaflet's resuspension onto the neo-PV annulus.

onto the neo-PV annulus. A leaflet patch extension before resuspension was necessary in 7 patients $(58 \%)$ ). The postoperative right ventricular pressure was $30 \%$ of systemic pressure in 1 patient $(8 \%), 50 \%$ in 5 patients $(42 \%)$, and $75 \%$ in 6 patients $(50 \%)$.

Median intensive care unit stay and hospitalization were 3 days (IQR, 3-4 days) and 13 days (IQR, 9-16 days), respectively. At discharge, median peak RVOT gradient was $30 \mathrm{~mm} \mathrm{Hg}$ (IQR, 24-40 mm Hg), and the degree of PV regurgitation was none/mild in 11 patients $(92 \%)$ and moderate in 1 patient $(8 \%)$.

At a median follow-up time of 2.8 years (IQR, 2.33.2 years), none of the patients required reoperation or

\section{Intraoperative PV balloon dilation}

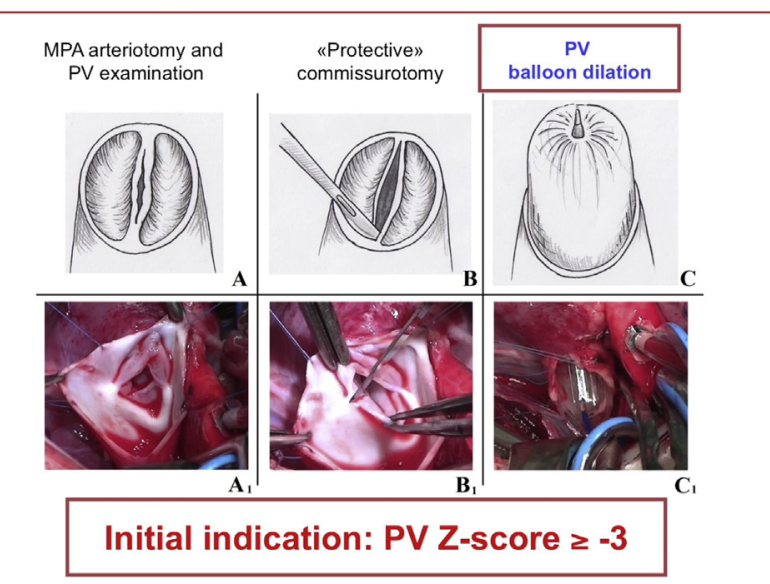

VIDEO 1. Surgical evolution of the application of PV-sparing procedures during repair of ToF. Video available at: https://www.jtcvs.org/article/ S0022-5223(19)30282-X/fulltext. hemodynamic reintervention. Median peak RVOT gradient was $35 \mathrm{~mm} \mathrm{Hg}$ (IQR, 30-40 $\mathrm{mm} \mathrm{Hg}$ ); the median PV annulus Z-score was -0.5 (IQR, $-0.6-0$ ), and the degree of PV regurgitation was none/mild in 10 patients $(83 \%)$ and moderate in 2 patients $(17 \%)$.

\section{CONCLUSIONS}

At the beginning of our experience with PV preservation during ToF repair, we were selecting our patients by treating the milder forms with a PV Z score -3 or greater by intraoperative balloon PV dilation. ${ }^{1}$ We progressively extended the surgical indications by introducing additional surgical maneuvers (eg, a leaflet PV delamination plasty) ${ }^{2-4}$ for achieving a satisfactory PV competence even in severe forms ( $\mathrm{PV} \mathrm{Z}$ scores $<-3$ ). In patients with $\mathrm{ToF}$ and marked PV annulus hypoplasia (PV Z-score $\leq 4$ ), intraoperative balloon PV dilation can disrupt the integrity of the PV annulus (due to excessive stretching of the elastic fibers). A patch augmentation of the PV annulus allows an adequate enlargement of the PV area for resecting the subpulmonary muscle tissue and recreating a commissure to resuspend the delaminated leaflet tissue. With this technique, we were able to increase the number of patients who were candidates for $\mathrm{PV}$ preservation during TOF repair by adding more severe forms (Z-scores <-4) and patients with unicommisural PVs, which was a contraindication to our initial strategy of repair. A longer follow-up is needed.

\section{References}

1. Vida VL, Padalino MA, Maschietto N, Biffanti R, Anderson RH, Milanesi O, et al. The balloon dilation of the pulmonary annulus during early repair of tetralogy of Fallot. Catheter Cardiovasc Interv. 2012;80:915-21. 
2. Vida VL, Guariento A, Castaldi B, Sambugaro M, Padalino MA, Milanesi O, et al. Evolving strategies for preserving the pulmonary valve during early repair of tetralogy of Fallot: mid-term results. J Thorac Cardiovasc Surg. 2014;147:687-96.

3. Vida VL, Angelini A, Guariento A, Frescura C, Fedrigo M, Padalino M, et al. Preserving the pulmonary valve during early repair of tetralogy of Fallot: anatomic substrates and surgical strategies. J Thorac Cardiovasc Surg. 2015; 149:1358-63.e1.

4. Vida VL, Zucchetta F, Stellin G. Pulmonary valve-sparing techniques during repair of tetralogy of Fallot: the delamination plasty (video). J Thorac Cardiovasc Surg. 2016;151:1757-8. 